\title{
The Parkinson Disease Protein Leucine-Rich Repeat Kinase 2 Transduces Death Signals via Fas-Associated Protein with Death Domain and Caspase-8 in a Cellular Model of Neurodegeneration
}

\author{
Cherry Cheng-Ying Ho, ${ }^{1,2}$ Hardy J. Rideout, ${ }^{2}$ Elena Ribe, ${ }^{1}$ Carol M. Troy, ${ }^{1,2,4}$ and William T. Dauer ${ }^{2,3}$ \\ Departments of ${ }^{1}$ Pathology, ${ }^{2}$ Neurology, and ${ }^{3}$ Pharmacology, and ${ }^{4}$ Taub Center for the Study of Alzheimer's Disease and the Aging Brain, Columbia \\ University, New York, New York 10032
}

\begin{abstract}
Neurodegenerative illnesses such as Parkinson and Alzheimer disease are an increasingly prevalent problem in aging societies, yet no therapies exist that retard or prevent neurodegeneration. Dominant missense mutations in leucine-rich repeat kinase 2 (LRRK2) are the most common genetic cause of Parkinson disease (PD), but the mechanisms by which mutant forms of LRRK2 disrupt neuronal function and cause cell death remain poorly understood. We report that LRRK2 interacts with the death adaptor Fas-associated protein with death domain (FADD), and that in primary neuronal culture LRRK2-mediated neurodegeneration is prevented by the functional inhibition of FADD or depletion of caspase-8, two key elements of the extrinsic cell death pathway. This pathway is activated by disease-triggering mutations, which enhance the LRRK2-FADD association and the consequent recruitment and activation of caspase-8. These results establish a direct molecular link between a mutant PD gene and the activation of programmed cell death signaling, and suggest that FADD/caspase-8 signaling contributes to LRRK2-induced neuronal death.
\end{abstract}

Key words: Parkinson's disease; apoptosis; neuronal apoptosis; neuronal death; neuron death; caspase

\section{Introduction}

Parkinson disease (PD) is characterized by motor and cognitive dysfunction reflecting widespread neurodegeneration, particularly of midbrain dopaminergic neurons. PD most commonly presents as a sporadic illness, but over the past decade insights into the molecular mechanisms of PD-related neurodegeneration have emerged from the discovery of mutations underlying rare inherited forms of the disease (Cookson et al., 2005). Despite these advances, the key signaling events that cause neurodegeneration in PD remain poorly defined.

One set of signaling pathways that can elicit cell death in response to cellular insults such as an accumulation of misfolded proteins, or oxidative stress (both of which have been implicated

\footnotetext{
Received 0ct. 26, 2008; revised Nov. 17, 2008; accepted Dec. 16, 2008.

This work was supported by National Institutes of Neurological Disorders and Stroke Grants K02 NS045798 and R01 NS061098, the Parkinson Disease Foundation, and the Anne and Bernard Spitzer Center for Cell and Genetic Therapy for Parkinson Disease. We thank the following people for their helpful comments on this manuscript: Serge Przedborski, Jonathan Javitch, Gilbert DiPaolo, Chris Henderson, and members of the Dauer Laboratory. Thanks also to Kana Tsukamoto for outstanding technical assistance. We also thank Drs. Dennis Dickson and Zbigniew Wszolek of the Mayo Clinic (Jacksonville, FL) for providing LRRK2-positive human brain tissue, Dr. Jean-Paul Vonsattel of the New York Brain Bank for providing control and idiopathic PD brain tissue, and Dr. Lorraine Clarke of Columbia University for genotyping idiopathic PD samples. The plasmid for caspase-8 was a kind gift from Dr. Junying Yuan the plasmid for LZ-FADD-DD was a kind gift from Dr. Milton Werner; the plasmid for death receptor 3 was a kind gift from Dr. Shie-Liang Hsieh; the LRRK2 antibody was a kind gift from Dr. Zhenyu Yue; the anti-mouse FADD antibody was a kind gift from Dr. Andreas Strasser.

Correspondence should be addressed to William Dauer, Columbia University, 650 West 168th Street, Room 310, New York, NY 10032. E-mail: wtd3@columbia.edu.

DOI:10.1523/JNEUROSCI.5175-08.2009

Copyright $\odot 2009$ Society for Neuroscience $\quad$ 0270-6474/09/291011-06\$15.00/0
}

in PD), are those of programmed cell death. The core of these pathways is composed of proteolytic caspases that, when activated, lead to a highly regulated process of cell death. Two broadly defined pathways can trigger programmed cell death: the intrinsic pathway, which is controlled by factors that are released by mitochondria and activate caspase- 9 , and the extrinsic pathway, which is typically initiated by cell surface "death receptors" such as tumor necrosis factor receptors (TNF-R) and Fas that lead to caspase-8 activation via the death adaptor Fas-associated protein with death domain (FADD). Whereas most studies have addressed the potential involvement of the intrinsic pathway in PD-related neurodegeneration, activated caspase- 8 has been observed in postmortem PD brain tissue (Hartmann et al., 2001), and modulation of the extrinsic pathway can reduce dopamine neuron loss in the MPTP mouse model of PD (Dauer and Przedborski, 2003; Hayley et al., 2004; McCoy et al., 2006). Moreover, extrinsic pathway signals are crucial mediators of inflammation, which is also postulated to mediate neurotoxicity in PD (Hirsch et al., 2005).

Dominantly inherited missense mutations in leucine-richrepeat kinase 2 (LRRK2) are the most common genetic cause of $\mathrm{PD}$, causing a clinical syndrome that is clinically and pathologically indistinguishable from sporadic PD. LRRK2 contains both GTPase and kinase signaling domains, as well as LRR and WD40 protein-protein interaction domains (Fig. $1 \mathrm{~A}$ ). Many potentially pathogenic sequence alterations have been identified in LRRK2 (Goldwurm et al., 2005), but five missense mutations (Fig. 1A) 
clearly segregate with PD in large family studies (Bonifati, 2007). Two of these mutations (R1441G, R1441C) are located in the GTPase domain (termed Ras of complex proteins, or "Roc" domain), a third (Y1699C) falls in a region between the GTPase and kinase domains (termed the C-terminal of Roc, or "COR" domain), and two other mutations (G2019S and I2020T) are in the kinase domain (Fig. $1 A)$. Whereas these mutations may affect LRRK2 GTPase (Guo et al., 2007; Lewis et al., 2007; Li et al., 2007) and kinase (West et al., 2005; Greggio et al., 2006; Smith et al., 2006; West et al., 2007) activity, published studies differ as to whether other mutations in LRRK2 significantly alter its kinase function (West et al., 2005; Gloeckner et al., 2006; Greggio et al., 2006; MacLeod et al., 2006; Smith et al., 2006; West et al., 2007). LRRK2 induces apoptotic neuronal death (Iaccarino et al., 2007) that requires intact kinase function (Greggio et al., 2006; Smith et al., 2006), but physiologically relevant LRRK2 substrates or downstream effectors have yet to be identified.

We demonstrate in vitro that LRRK2 interacts with the death adaptor protein FADD, and that this interaction recruits and activates caspase-8-dependent neuronal death. Moreover, the potential role of the extrinsic cell death pathway in PD is supported by findings in postmortem brain tissue of patients with LRRK2associated Parkinson disease.

\section{Materials and Methods}

Cloning of human LRRK2 cDNA. A human LRRK2 cDNA was amplified and fully sequenced from HEK cell cDNA and the translated amino acid sequence conformed to human LRRK2 AAI17181 in the NCBI database. All subsequent mutations were generated using site-directed mutagenesis and all mutant clones were resequenced to confirm their accuracy.

Plasmids. LRRK2 cDNA from HEK 293 cells was cloned in pcDNA-DEST53 (Invitrogen). Cytoplasmic domains of TNFR1, TNFR3, tumor necrosis factor-related apoptosis-inducing ligand receptor (TRAIL-R) 1, TRAIL-R2, and Fas were cloned in pcDNA27, whereas FADD, TRADD, RIP1, and RAIDD cDNA were cloned in pcDNA3.1/nV5-DEST (Invitrogen). All subsequent mutants were generated using sitedirected mutagenesis, and all mutant clones were resequenced to confirm their accuracy.

Cell lines and primary neuronal cultures. CAD cells were grown in DMEM/F12 (Invitrogen) supplemented with $8 \%$ fetal bovine serum. 293 cells were grown in DMEM (Invitrogen) with $10 \%$ serum. CAD cells were transfected with Lipofectamine/PLUS, whereas 293T cells were transfected with Lipofectamine 2000 or Lipofectamine LTX (Invitrogen). Cultures of cortical neurons from E16 mice were maintained in Neurobasal medium containing B-27 supplements (Invitrogen), and transfected with Lipofectamine $20004 \mathrm{~d}$ after being plated. Primary neurons immunoblotted as in D.
A
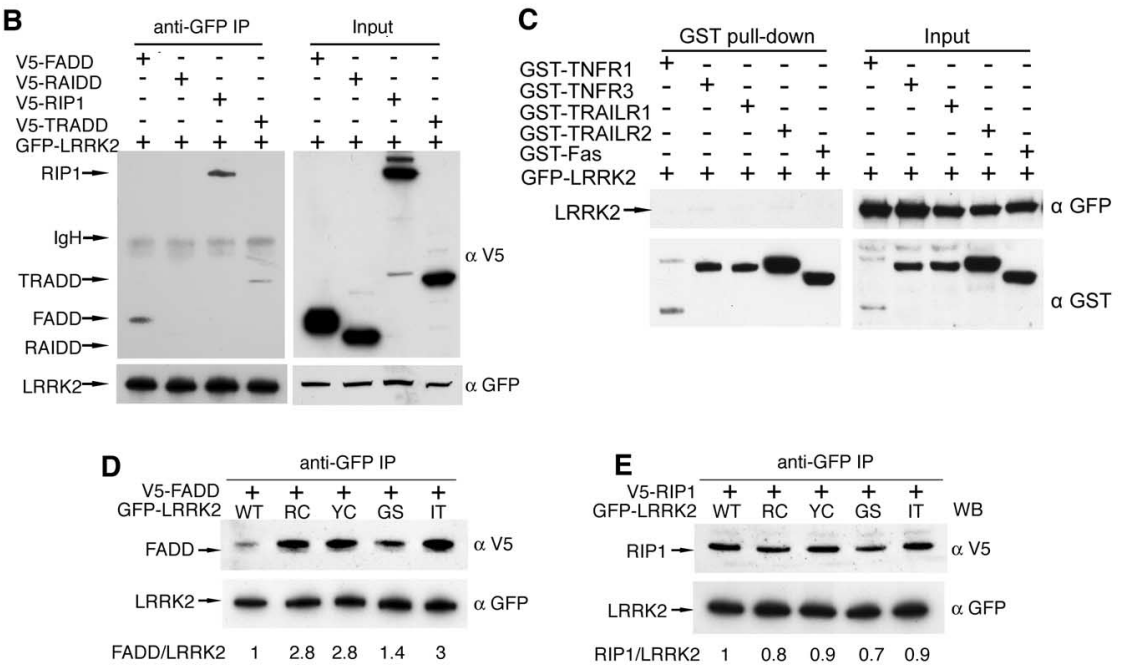

Input
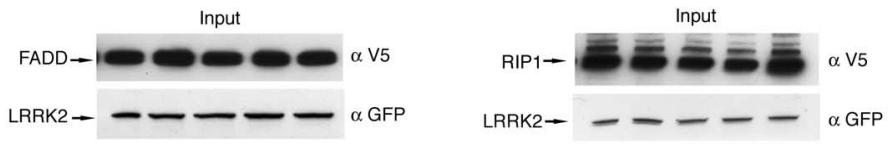

$\mathbf{F}$ G

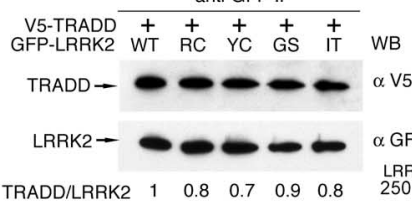

H

H V5-FADD $\frac{\text { anti-GFP IP }}{2++++t}$ GFP-LRRK2 WT KD RC/KD YC/KD GS/KD IT/KD FADD $\rightarrow-\infty-\infty+\alpha$ V5 LRRK2 $\rightarrow-\infty \Rightarrow+\alpha$ GFP $\begin{array}{lllllll}\text { FADD/LRRK2 } & 1 & 1 & 0.8 & 1.3 & 0.8 & 0.8\end{array}$ $\begin{array}{llll}0.7 & 0.9 & 0.8 & 250 \mathrm{kD} \\ \mathrm{FADD} & & & \end{array}-\alpha_{\text {FADD }}$ Input $\quad 25 \mathrm{kD}$ Input
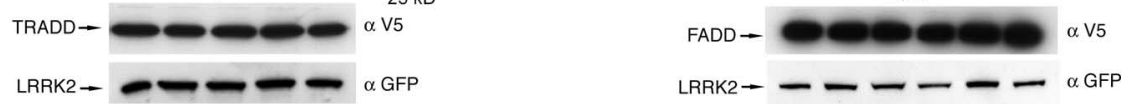

Figure 1. Parkinson disease mutations enhance the interaction between LRRK2 and FADD. $\boldsymbol{A}$, Domain structure and Parkinson disease mutations of LRRK2. LRR, leucine-rich repeat; Roc, Ras of complex GTPase; COR, C-terminal of Roc. Five dominantly inherited PD-causing missense mutations are indicated. $\boldsymbol{B}$, LRRK2 interacts with death adaptor proteins of the extrinsic pathway. 293T cells coexpressing GFP-LRRK2 and V5-tagged death adaptor proteins were subjected to anti-GFP immunoprecipitation followed by anti-GFP and anti-V5 immunoblotting. C, LRRK2 does not associate with death receptors. 293T cells coexpressing GFP-LRRK2 with GST-tagged cytoplasmic domains of death receptors were subjected to GST pull-down followed by immunoblotting. D, Enhanced association of FADD with LRRK2 PD mutants. 293T cells were cotransfected with wild-type (WT) or PD mutant GFP-LRRK2 and V5-tagged FADD. Anti-GFP immunoprecipitates were analyzed by anti-V5 and anti-GFP immunoblots. Ratios indicate the binding of FADD to LRRK2 relative to WT-LRRK2. E, PD mutations fail to enhance the association of LRRK2 with RIP1. 293T cells were cotransfected with V5-tagged RIP1 and WT or PD mutant GFP-LRRK2 and were assessed as in D. F, PD mutations fail to enhance the association of LRRK2 with TRADD. 293T cells were cotransfected with V5-tagged TRADD and WT or PD mutant GFP-LRRK2 and were assessed as in D. G, Endogenous LRRK2-FADD complex formation in mouse brain. Whole-brain lysates from 1-year-old wild-type mice were subjected to immunoprecipitation with anti-FADD (clone 7A2). Copurified LRRK2 was determined with anti-LRRK2 immunoblotting. $\boldsymbol{H}$, Blocking LRRK2 kinase function prevents the enhanced FADD association with LRRK2 disease mutants. 293T cells expressing V5-tagged FADD and GFP-tagged WT or kinase-dead (KD) LRRK2 were immunoprecipitated and

were transfected with LRRK2 expression constructs and pCMS-EGFP (Clontech) at 10:1 ratio. In cotransfection experiments, LRRK2 and FADD-DD or LZ-FADD-DD expression constructs were used at a 2:1 ratio. Each experiment was performed on coverslips in triplicate, at least three times, and $>100$ cells/coverslip were quantified. Apoptotic neurons were defined as cells having two or more condensed apoptotic nuclear bodies visualized using DAPI.

Antibodies. Mouse anti-glutathione S-transferase (GST) clone GST-2 and anti-FLAG M2 were purchased from Sigma. Mouse anti-green fluorescent protein (GFP) was from Roche. Rabbit anti-GFP was from Ab- 
A

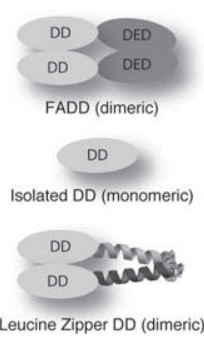

B

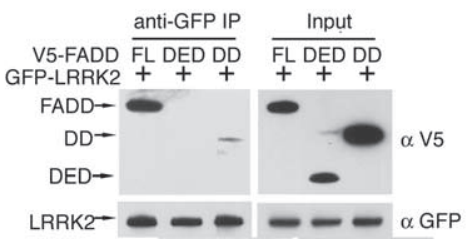

D

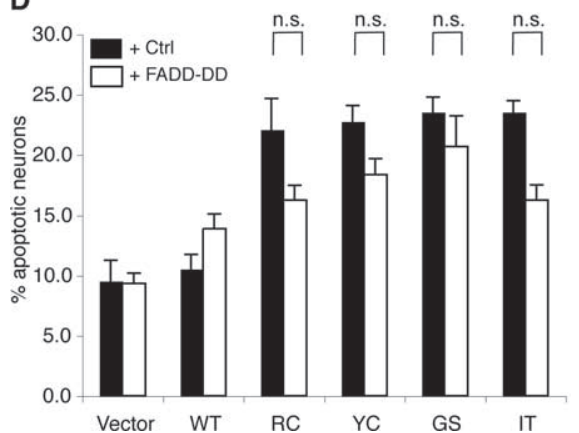

C

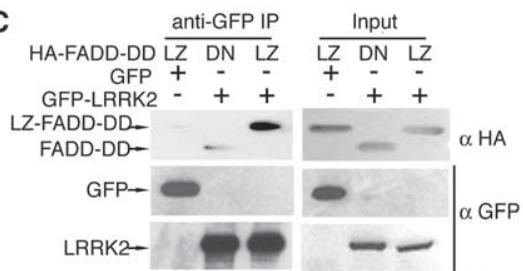

E

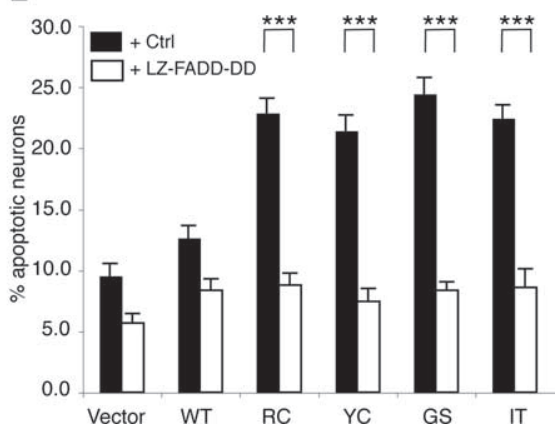

Figure 2. $\quad$ LRRK2-induced neuronal death requires FADD. $\boldsymbol{A}$, A schematic depicts the domain structure of FADD, the isolated death domain (FADD-DD), and the leucine-zipper-DD (LZ-FADD-DD) in which the death domain is dimerized through the addition of a leucine zipper. $\boldsymbol{B}$, FADD interacts with LRRK2 via its DD. GFP-LRRK2 was coexpressed with V5-tagged full-length FADD, FADD-DED, or FADD-DD in 293T cells, and was immunoprecipitated with anti-GFP. Copurified FADD or FADD domains were detected by anti-V5 immunoblotting. $C$, LRRK2-FADD interaction is enhanced by dimerization of FADD-DD. The interaction between GFP-LRRK2 and monomeric (DD) or dimeric (LZ) FADD-DD was assessed by anti-HA after immunoprecipitation with antiGFP in 293 T cells. D, FADD-DD is a poor inhibitor of LRRK2 neurotoxicity. Mouse cortical neurons were transfected with LRRK2 + lacZ (Ctrl) or LRRK2 + FADD-DD. A GFP reporter was cotransfected in each case. Transfected neurons displaying apoptotic nuclear morphology were counted $48 \mathrm{~h}$ after transfection using DAPI. Data are the mean \pm SEM from three individual experiments of triplicate coverslips (n.S., nonsignificant; ANOVA with Tukey's post hoc test). $\boldsymbol{E}$, Dimeric FADD-DD effectively blocks LRRK2 neurotoxicity. Mouse cortical neurons expressing LRRK + lacZ (Ctrl) or LRRK2 + LZ-FADD-DD were assessed as in $\boldsymbol{D}\left({ }^{* * *} p<0.001\right)$.

cam. Mouse anti-V5 was from Invitrogen. Mouse anti-FADD was from BD transduction. Rat anti-FADD clone 7A2 was a gift from A. Strasser. Rabbit anti-mouse LRRK2 was a gift from Z. Yue (Li et al., 2007). Mouse anti-HA clone F-7 and rabbit anti-caspase- 1 were from Santa Cruz Biotechnology. Mouse anti-caspase- 8 clone $1 \mathrm{C} 12$ and rabbit anti-human caspase- 9 were from Cell Signaling Technology. Mouse anti-caspase- 8 clone $\mathrm{C} 15$ was from Alexis. Rabbit anti-caspase- 8 and rabbit anticaspase- 9 were from MBL.

Immunofluorescent labeling. Forty-eight hours after transfection, formaldehyde-fixed neurons on coverslips were blocked in PBS containing $0.25 \%$ Triton X-100 and 5\% normal donkey serum for $30 \mathrm{~min}$. Coverslips were then incubated overnight at $4^{\circ} \mathrm{C}$ in rabbit anti-GFP antibodies diluted in block solution. The next day coverslips were washed, incubated with FITC-conjugated secondary antibodies, and washed in PBS before mounting using Vectashield Mounting Media with DAPI (Vector Laboratories). Immunostained neurons were then subjected to quantification for apoptosis.

GST-pulldown and coimmunoprecipitation analysis. 293T cells transfected with various expression constructs were Dounce homogenized in lysis buffer (20 mm HEPES, pH 7.4, 150 mm NaCl, 0.1-0.5\% NP-40, 2 mM EGTA, 2 mм MgCl2, 10\% glycerol, 1 mm sodium orthovanadate, 10 $\mathrm{mm} \mathrm{NaF}, 25 \mathrm{~mm} \beta$-glycerophosphate, $\mathrm{pH}$ 7.2, and protease inhibitors). After centrifugation and preclearing, lysates were incubated with glutathione affinity gel (Sigma) or rabbit anti-GFP antibody with protein-A agarose for $3 \mathrm{~h}$ to overnight. The immunocomplexes were washed five times with isotonic or hypertonic lysis buffer $(250 \mathrm{~mm} \mathrm{NaCl})$ and released from beads by boiling in $1 \times$ Laemmli sample buffer for immunoblot analysis.

RNA interference. Penetratin1 (Pen 1 ) coupled small interfering RNA (siRNA) were generated as described previously (Davidson et al., 2004). The target sequences used were as follows: caspase-8 GCACAGAGAGAAGAAUGAG; caspase-9 GGCACCCUGGCUUCACUCU. Three days after plating, primary neurons were treated with $350 \mathrm{~nm}$ Pen1-siRNA for $24 \mathrm{~h}$. Cells were transfected and $48 \mathrm{~h}$ later, assessed for apoptotic death as described above.

\section{Results}

The kinase domain of LRRK2 is most closely related to that of LRRK1, followed by the receptor interacting protein (RIP) family of serine/threonine kinases (Manning et al., 2002), crucial regulators of cell survival and death (Meylan and Tschopp, 2005; Festjens et al., 2007). RIP1, the best studied member of this family, transduces signals downstream of death receptors (e.g., TNF $\alpha$, Fas ligand, TRAIL) of the extrinsic cell death pathway. This led us to explore whether LRRK2 might also participate in extrinsic cell death signaling.

We first assessed whether, like RIP1, LRRK2 can interact with death adaptor proteins of the extrinsic cell death pathway. Coimmunoprecipitation (co-IP) experiments demonstrated that LRRK2 interacts with FADD and TRADD, two key death adaptor proteins. FADD, TRADD and RIP1 all contain death domains (DD), and LRRK2 also copurified with RIP1. However, LRRK2 did not bind the DDcontaining protein RAIDD that is not implicated in extrinsic apoptotic signaling (Fig. $1 B$ ), nor did it bind any of the DDcontaining death receptors (TNF-R1, TNF-R3, Fas, TRAIL-R1, TRAIL-R2) (Fig. 1C). These data suggest that LRRK2 specifically interacts with a subset of DD-containing proteins that transduce extrinsic cell death signals.

To explore the potential disease relevance of these LRRK2interacting proteins, we next tested whether their interaction with LRRK2 was altered by PD-linked mutations. We found that all mutations tested enhanced the interaction between LRRK2 and FADD (Fig. 1C). In contrast, PD mutations had no effect on the association of LRRK2 with RIP1 or TRADD (Fig. 1D,E). These findings led us to determine whether these proteins interact in brain tissue, and LRRK2 did copurify with FADD in mouse brain lysates (Fig. $1 F$ ). Blocking LRRK2 kinase function prevents the death of primary neurons transfected with PD mutant forms of LRRK2 (Greggio et al., 2006; Smith et al., 2006; data not shown), so we next explored whether blocking kinase function also alters FADD binding. Indeed, the kinase-deficient LRRK2 mutation (K1906R) normalized the enhanced FADD binding by caused by PD mutations (Fig. $1 G$ ), consistent with a potential role for this death adaptor protein in LRRK2 neurotoxicity. These data led us to hypothesize that FADD may be recruited to LRRK2, 
leading to the formation of a complex similar to the death inducing signaling complex (DISC) formed by Fas, FADD and other signaling proteins.

To further explore this notion, we characterized the interaction between LRRK2 and FADD. FADD transduces death signals by binding to ligand-activated Fas via its DD and recruiting and activating caspase- 8 via its death-effector domain (DED) (Fig. 2A). FADD dimerizes on binding to Fas, a crucial event that greatly enhances both the FADD-Fas interaction and caspase- 8 activation. Similarly, in co-IP studies we found that FADD binds to LRRK2 via its DD, yet the interaction was weak, despite the high expression level of this isolated domain (Fig. 2 B). Mimicking the dimeric conformation of physiologically active FADD-DD by attaching a leucine-zipper domain (LZ; LZ-FADDDD) restored its interaction with LRRK2 (Fig. 2C). LZ-FADD-DD and the isolated FADD-DD are dominant negative inhibitors of FADD signaling because they lack the DED necessary to recruit caspase- 8 . Thus, we used these molecules to ask whether blocking FADD function affects LRRK2-induced neuronal death. The monomeric FADD-DD failed to significantly suppress the neuronal death caused by LRRK2 PD mutants, although there was a trend for death suppression (Fig. 2D). In contrast, dimeric LZ-FADD-DD completely blocked LRRK2-mediated neurodegeneration (Fig. $2 E$ ). Thus, the strength of FADD-LRRK2 interaction (Fig. $2 B, C$ ) correlates with the ability of the dominant negative FADD mutant to block LRRK2induced cell death (Fig. 2D,E), further supporting a functional relationship between these proteins.

These data suggest that the LRRK2FADD interaction may serve to recruit and activate caspase- 8 . In the absence of exogenous FADD, only a trace amount of caspase-8 copurified with LRRK2 (Fig. $3 A$ ).

The expression of exogenous FADD significantly increased the amount of caspase- 8 that copurified with LRRK2, suggesting that LRRK2, FADD, and caspase- 8 are components of a multiprotein complex (Fig. 3A). To evaluate the function of caspase- 8 in transducing the LRRK2-induced death signal, we reduced endogenous caspase- 8 levels in neurons by RNA interference (RNAi). The efficiency and the specificity of this caspase- 8 siRNA have been previously established in primary neuronal cultures (Davidson et al., 2004) and is effective against Fas-mediated neuronal death (Carol Troy, personal communication). Knocking down caspase-8 significantly reduced LRRK2-induced neurodegeneration (Fig. 3B). In contrast, RNAi knockdown of caspase-9, which transduces signals in the intrinsic apoptotic pathway, did not significantly attenuate LRRK2-induced neuronal death (Fig. 3C) (this siRNA is effective against HNE-induced neuronal death; Carol Troy, personal communication).
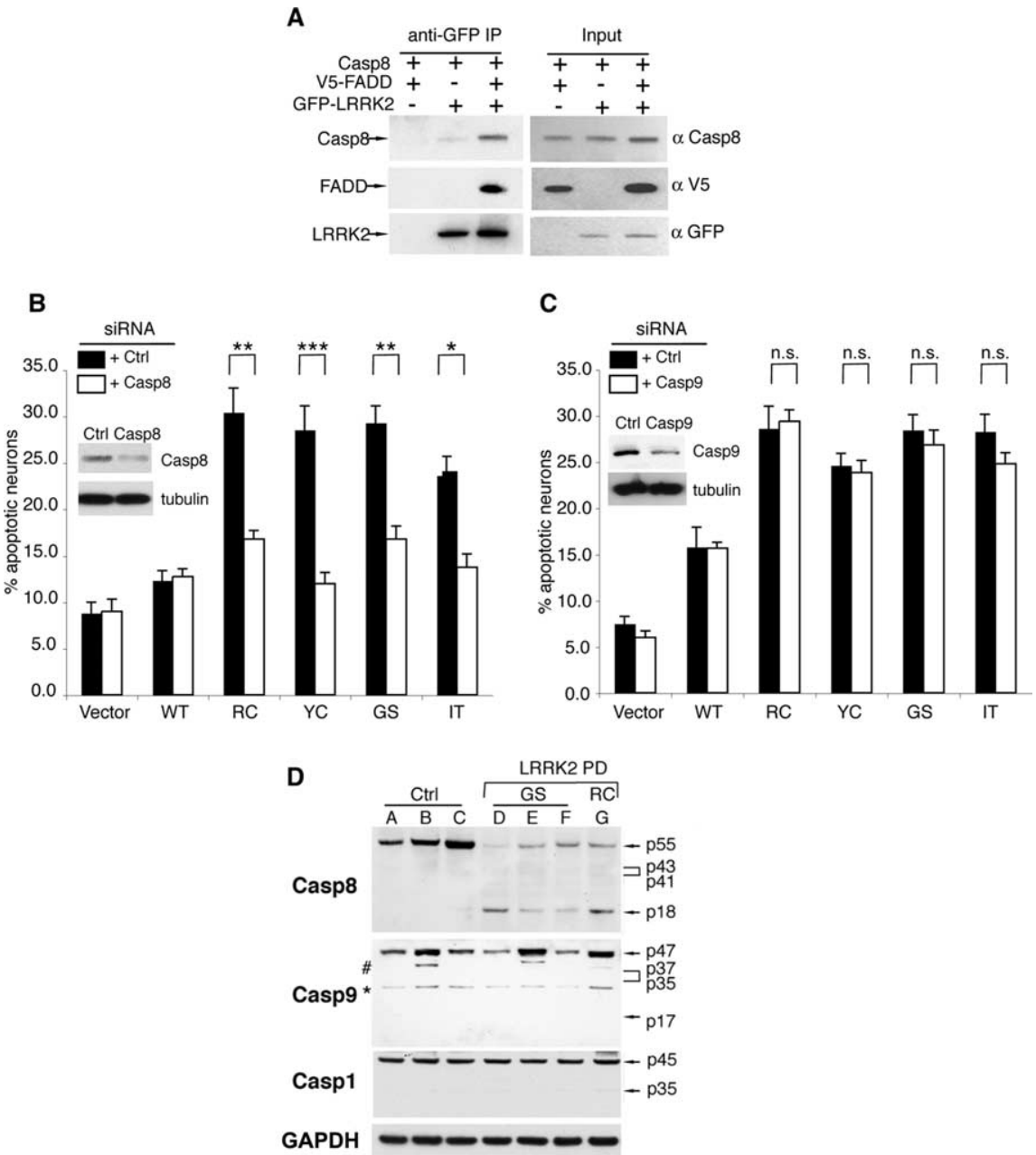

Figure 3. LRRK2-induced neuronal death is caspase-8 (Casp8) dependent. $\boldsymbol{A}$, FADD recruits caspase-8 to LRRK2. $293 \mathrm{~T}$ cells were transfected with GFP-LRRK2, V5-FADD, and caspase-8 inactive mutant (C360S), as indicated. GFP-LRRK2 was immunoprecipi列 8 siRNA $24 \mathrm{~h}$ before transfection with GFP-tagged wild-type (WT) or mutant LRRK2. Data are the mean \pm SEM from three (aspase-9 RNAi, transfection, and neuronal death were performed and assessed as in B. D. Caspase-8 is

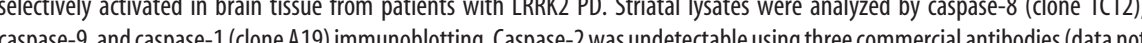
shown). The locations of the pro-caspase isoforms and their corresponding cleavage products are indicated ( ${ }^{*}$ nonspecific immunoreactive bands; " potential cleavage products with higher than expected mass: 37 and $35 \mathrm{kDa}$ ).

Our results suggest that caspase- 8 activation may be a pathogenic event in PD patients with LRRK2 mutations. To further test this notion, we measured whether caspase- 8 is activated in brain lysates from control subjects and PD patients with LRRK2 mutations. Caspase- 8 is activated by homodimerization (Boatright et al., 2003), which also leads to autoproteolytic processing of the enzyme into multiple smaller species $(43,41,18$, and $10 \mathrm{kDa}$; the $10 \mathrm{kDa}$ fragment is not detected by the commercial antibodies $1 \mathrm{C} 12$ or C15). The presence of one or more of these cleavage products was found in all patients with LRRK2 mutations (Fig. $3 D$, supplemental Table 1, available at www.jneurosci.org as supplemental material). One of two patients tested with idiopathic disease (i.e., no LRRK2 mutation) also showed low level caspase- 8 activation (data not shown); more cases will be required to determine the fraction of idiopathic PD that may show activation of the extrinsic pathway. Processing of caspase- 8 can 
lead to decreased levels of the full-length enzyme (Yang et al., 1998), and we also found markedly decreased levels of procaspase- 8 in the PD patients with LRRK2 mutations. In contrast, we did not observe a clear pattern of activation for the apical caspases that control the intrinsic pathway (caspase-9) or inflammation-related death signaling (caspase-1) (Fig. 3D).

\section{Discussion}

Our findings link a PD-causing gene directly to the activation of a cell death signaling pathway, and we provide direct support for this notion with evidence from human postmortem tissue from PD patients bearing two different LRRK2 mutations. We demonstrate that PD-linked mutations enhance the interaction of LRRK2 with FADD, leading to the recruitment and activation of caspase-8. Our data also show that blocking LRRK2 kinase function eliminates the increased FADD binding caused by PD mutations, providing a potential mechanism for how this mutation blocks LRRK2-mediated neuronal death.

Multiple lines of evidence support the notion that apoptotic machinery contributes to neurodegeneration in PD. For example, apoptotic nuclei have been identified in DA neurons of the substantia nigra, and many reports have documented altered levels or activation of key apoptotic molecules in PD postmortem tissue (Vila and Przedborski, 2003). Nevertheless, a longstanding question about such data is whether they indicate a key role for cell death signaling pathways, or reflect a late downstream consequence of PD-related cellular demise. Our data suggest that, at least in patients with LRRK2 mutations, activation of the apoptotic cascade may be an important early event in disease pathogenesis. The significance of these findings is highlighted by the fact that LRRK2 is the most common genetic cause of PD, but future work is necessary to determine the relevance of our findings to idiopathic PD, in which a role for LRRK2 is less clearly defined.

Two studies have implicated LRRK2 in the control of neurite morphology (MacLeod et al., 2006; Plowey et al., 2008) and, interestingly, many reports document the presence of cell death signaling molecules in neurites and synaptic terminals (Mattson et al., 1998; Chung et al., 2003; Cowan and Roskams, 2004; Carson et al., 2005). These studies indicate that caspase activation can occur locally within neuronal processes, leading to process degeneration. Particularly relevant to our findings is the study of Carson et al. (2005), who reported that caspase- 8 is activated in presynaptic terminals in response to a deafferentated lesion, and is subsequently retrogradely transported to the cell body where it eventually triggers apoptotic cell death. Moreover, a number of extrinsic pathway-related molecules are concentrated within neuronal processes, and this pathway can modulate neuronal process morphology and synaptic function (Meffert et al., 2003; Boulanger and Shatz, 2004; Ma et al., 2006; Stellwagen and Malenka, 2006; Heckscher et al., 2007). Thus, our data raise the possibility that FADD/caspase- 8 signaling may contribute to the neuritic pathology associated with LRRK2, and that this process leads to retrograde signaling that ultimately culminates in cell body death. Indeed, such a scenario would be consistent with studies of human PD, suggest that the midbrain dopaminergic neuron degeneration begins in striatal terminal projections and is later followed by cell body death in the substantia nigra (Scherman et al., 1989; Fearnley and Lees, 1991; Lee et al., 2000).

\section{References}

Boatright KM, Renatus M, Scott FL, Sperandio S, Shin H, Pedersen IM, Ricci JE, Edris WA, Sutherlin DP, Green DR, Salvesen GS (2003) A unified model for apical caspase activation. Mol Cell 11:529-541.
Bonifati V (2007) LRRK2 low-penetrance mutations (Gly2019Ser) and risk alleles (Gly2385Arg)-linking familial and sporadic Parkinson disease. Neurochem Res 32:1700-1708.

Boulanger LM, Shatz CJ (2004) Immune signalling in neural development, synaptic plasticity and disease. Nat Rev Neurosci 5:521-531.

Carson C, Saleh M, Fung FW, Nicholson DW, Roskams AJ (2005) Axonal dynactin p150Glued transports caspase- 8 to drive retrograde olfactory receptor neuron apoptosis. J Neurosci 25:6092-6104.

Chung CW, Hong YM, Song S, Woo HN, Choi YH, Rohn T, Jung YK (2003) Atypical role of proximal caspase- 8 in truncated Tau-induced neurite regression and neuronal cell death. Neurobiol Dis 14:557-566.

Cookson MR, Xiromerisiou G, Singleton A (2005) How genetics research in Parkinson disease is enhancing understanding of the common idiopathic forms of the disease. Curr Opin Neurol 18:706-711.

Cowan CM, Roskams AJ (2004) Caspase-3 and caspase-9 mediate developmental apoptosis in the mouse olfactory system. J Comp Neurol 474:136-148

Dauer W, Przedborski S (2003) Parkinson disease: mechanisms and models. Neuron 39:889-909.

Davidson TJ, Harel S, Arboleda VA, Prunell GF, Shelanski ML, Greene LA, Troy CM (2004) Highly efficient small interfering RNA delivery to primary mammalian neurons induces MicroRNA-like effects before mRNA degradation. J Neurosci 24:10040-10046.

Fearnley JM, Lees AJ (1991) Ageing and Parkinson's disease: substantia nigra regional selectivity. Brain 114:2283-2301.

Festjens N, Vanden Berghe T, Cornelis S, Vandenabeele P (2007) RIP1, a kinase on the crossroads of a cell's decision to live or die. Cell Death Differ 14:400-410.

Gloeckner CJ, Kinkl N, Schumacher A, Braun RJ, O’Neill E, Meitinger T, Kolch W, Prokisch H, Ueffing M (2006) The Parkinson disease causing LRRK2 mutation I2020T is associated with increased kinase activity. Hum Mol Genet 15:223-232.

Goldwurm S, Di Fonzo A, Simons EJ, Rohé CF, Zini M, Canesi M, Tesei S, Zecchinelli A, Antonini A, Mariani C, Meucci M, Sacilotto G, Sironi F, Salani G, Ferreira J, Chien HF, Fabrizio E, Vanacore N, Dalla Libera A, Stocchi F, et al (2005) The G6055A (G2019S) mutation in LRRK2 is frequent in both early and late onset Parkinson disease and originates from a common ancestor. J Med Genet 42:e65.

Greggio E, Jain S, Kingsbury A, Bandopadhyay R, Lewis P, Kaganovich A, van der Brug MP, Beilina A, Blackinton J, Thomas KJ, Ahmad R, Miller DW, Kesavapany S, Singleton A, Lees A, Harvey RJ, Harvey K, Cookson MR (2006) Kinase activity is required for the toxic effects of mutant LRRK2/ dardarin. Neurobiol Dis 23:329-341.

Guo L, Gandhi PN, Wang W, Petersen RB, Wilson-Delfosse AL, Chen SG (2007) The Parkinson's disease-associated protein, leucine-rich repeat kinase 2 (LRRK2), is an authentic GTPase that stimulates kinase activity. Exp Cell Res 313:3658-3670.

Hartmann A, Troadec JD, Hunot S, Kikly K, Faucheux BA, Mouatt-Prigent A, Ruberg M, Agid Y, Hirsch EC (2001) Caspase-8 is an effector in apoptotic death of dopaminergic neurons in Parkinson disease, but pathway inhibition results in neuronal necrosis. J Neurosci 21:2247-2255.

Hayley S, Crocker SJ, Smith PD, Shree T, Jackson-Lewis V, Przedborski S, Mount M, Slack R, Anisman H, Park DS (2004) Regulation of dopaminergic loss by Fas in a 1-methyl-4-phenyl-1,2,3,6-tetrahydropyridine model of Parkinson disease. J Neurosci 24:2045-2053.

Heckscher ES, Fetter RD, Marek KW, Albin SD, Davis GW (2007) NF- $\kappa$ B, $\mathrm{I} \kappa \mathrm{B}$, and IRAK control glutamate receptor density at the Drosophila NMJ. Neuron 55:859-873.

Hirsch EC, Hunot S, Hartmann A (2005) Neuroinflammatory processes in Parkinson disease. Parkinsonism Relat Disord 11 [Suppl 1]:S9-S15.

Iaccarino C, Crosio C, Vitale C, Sanna G, Carrì MT, Barone P (2007) Apoptotic mechanisms in mutant LRRK2-mediated cell death. Hum Mol Genet 16:1319-1326.

Lee CS, Samii A, Sossi V, Ruth TJ, Schulzer m, Holden JE, Wudel J, Pal PK, de la Fuente-Fernandez R, Calne DB, Stoessl AJ (2000) In vivo positron emission tomographic evidence for compensatory changes in presynaptic dopaminergic nerve terminals in Parkinson's disease. Ann Neurol 47:493-503.

Lewis, PA, Greggio E, Beilina A, Jain S, Baker A, Cookson MR (2007) The R1441C mutation of LRRK2 disrupts GTP hydrolysis. Biochem Biophys Res Commun 357:668-671.

Li X, Tan YC, Poulose S, Olanow CW, Huang XY, Yue Z (2007) Leucine- 
rich repeat kinase 2 (LRRK2)/PARK8 possesses GTPase activity that is altered in familial Parkinson disease R1441C/G mutants. J Neurochem 103:238-247.

Ma Y, Li J, Chiu I, Wang Y, Sloane JA, Lu J, Kosaras B, Sidman RL, Volpe JJ, Vartanian T (2006) Toll-like receptor 8 functions as a negative regulator of neurite outgrowth and inducer of neuronal apoptosis. J Cell Biol 175:209-215.

MacLeod D, Dowman J, Hammond R, Leete T, Inoue K, Abeliovich A (2006) The familial Parkinsonism gene LRRK2 regulates neurite process morphology. Neuron 52:587-593.

Manning G, Whyte DB, Martinez R, Hunter T, Sudarsanam S (2002) The protein kinase complement of the human genome. Science 298:1912-1934.

Mattson MP, Keller JN, Begley JG (1998) Evidence for synaptic apoptosis. Exp Neurol 153:35-48.

McCoy MK, Martinez TN, Ruhn KA, Szymkowski DE, Smith CG, Botterman BR, Tansey KE, Tansey MG (2006) Blocking soluble tumor necrosis factor signaling with dominant-negative tumor necrosis factor inhibitor attenuates loss of dopaminergic neurons in models of Parkinson disease. J Neurosci 26:9365-9375.

Meffert MK, Chang JM, Wiltgen BJ, Fanselow MS, Baltimore D (2003) $\mathrm{NF}-\kappa \mathrm{B}$ functions in synaptic signaling and behavior. Nat Neurosci 6:1072-1078.

Meylan E, Tschopp J (2005) The RIP kinases: crucial integrators of cellular stress. Trends Biochem Sci 30:151-159.
Plowey ED, Cherra SJ 3rd, Liu YJ, Chu CT (2008) Role of autophagy in G2019S-LRRK2-associated neurite shortening in differentiated SH-SY5Y cells. J Neurochem 105:1048-1056.

Scherman D, Desnos C, Darchen F, Pollak P, Javoy-Agid F, Agid Y (1989) Striatal dopamine deficiency in Parkinson's disease: role of aging. Ann Neurol 26:551-557.

Smith WW, Pei Z, Jiang H, Dawson VL, Dawson TM, Ross CA (2006) Kinase activity of mutant LRRK2 mediates neuronal toxicity. Nat Neurosci 9:1231-1233.

Stellwagen D, Malenka RC (2006) Synaptic scaling mediated by glial TNF- $\alpha$. Nature 440:1054-1059.

Vila M, Przedborski S (2003) Targeting programmed cell death in neurodegenerative diseases. Nat Rev Neurosci 4:365-375.

West AB, Moore DJ, Biskup S, Bugayenko A, Smith WW, Ross CA, Dawson VL, Dawson TM (2005) Parkinson disease-associated mutations in leucine-rich repeat kinase 2 augment kinase activity. Proc Natl Acad Sci U S A 102:16842-16847.

West AB, Moore DJ, Choi C, Andrabi SA, Li X, Dikeman D, Biskup S, Zhang Z, Lim KL, Dawson VL, Dawson TM (2007) Parkinson diseaseassociated mutations in LRRK2 link enhanced GTP-binding and kinase activities to neuronal toxicity. Hum Mol Genet 16:223-232.

Yang X, Chang HY, Baltimore D (1998) Autoproteolytic activation of procaspases by oligomerization. Mol Cell 1:319-325. 